\title{
Recent developments in the nonelastic reaction code BRIEFF
}

\author{
H. Duarte
}

CEA/DAM/DIF, 91297 Arpajon, France

\begin{abstract}
The production of light-mass ion in nucleon induced reactions was lacking in the intranuclear cascade (INC) model of the nuclear reaction code BRIEFF. Deuteron candidates can now be created all along the evolution of the INC stage of reaction according a quasi-free coalescence process inside the nuclear+Coulomb field of the target nucleus. This process is partly based on the opposite process of the deuteron breakup already implemented. The emission of a deuteron candidate is the result of the competition between the deuteron formation and the deuteron breakup. This development of our INC model allows to calculate the production of deuteron in the full range of outgoing energy in nucleon induced reactions but also in deuteron induced reactions. Preliminary results of deuteron production in continuum in proton $(\mathrm{p}, \mathrm{xd})$ and deuteron $(\mathrm{d}, \mathrm{xd})$ induced reactions are presented and discussed.
\end{abstract}

\section{Introduction}

The Monte-Carlo code BRIEFF [1] is built on two stages: a fast stage described by the intranuclear cascade (INC) and a slower stage, the de-excitation of the compound nucleus formed at the end of the INC. The first versions of our intranuclear cascade code BRIC [2] calculated the observables from the products of the nonelastic nuclear reactions induced by nucleons and pions on nuclei at intermediate energy up to several $\mathrm{GeV}$. The reaction products in the INC stage were limited to the emission of these particles and to the compound nucleus while light-mass ions with $2 \leq \mathrm{A} \leq 4$ were emitted only in the evaporation of BRIEFF in more than the nucleons. BRIC has recently been extended to compute also non-elastic reactions induced by light-mass ions $\left(\mathrm{d}, \mathrm{t},{ }^{3} \mathrm{He}, \alpha\right.$-particle ...) [3]. However the emission of high-energy light-mass ions in the INC stage were still missing and the breakup of incident deuteron (and of any other ion with low binding energy) by the nuclear+Coulomb field of the target nucleus had to be improved.

We present how the deuterons are now included in our INC model as incident ions and also as emitted ions and we compare our results of double differential cross section of particle production with experimental data.

\section{Deuteron in BRIC}

The development of our INC model to compute deuteron induced reaction has been described in [3]. The deuteron density is now given by an Húlthen wave function and the nucleon potential in the deuteron center-of-mass $V_{d}^{*}$ is the nucleon-nucleon interaction in the ${ }^{1} S_{0}$ channel of Malfliet and Tjon [4]. The position and the energy-momentum of the deuteron in the target nucleus are updated by the equations of motion of the deuteron in the nuclear+Coulomb potential of the target then the positions and energy-momenta of the $n_{(d)}$ and $p_{(d)}$ are updated in the laboratory frame as it was done

\footnotetext{
a e-mail: helder.duarte@cea.fr
}

This is an Open Access article distributed under the terms of the Creative Commons Attribution-Noncommercial License 3.0, which permits unrestricted use, distribution, and reproduction in any noncommercial medium, provided the original work is properly cited. 


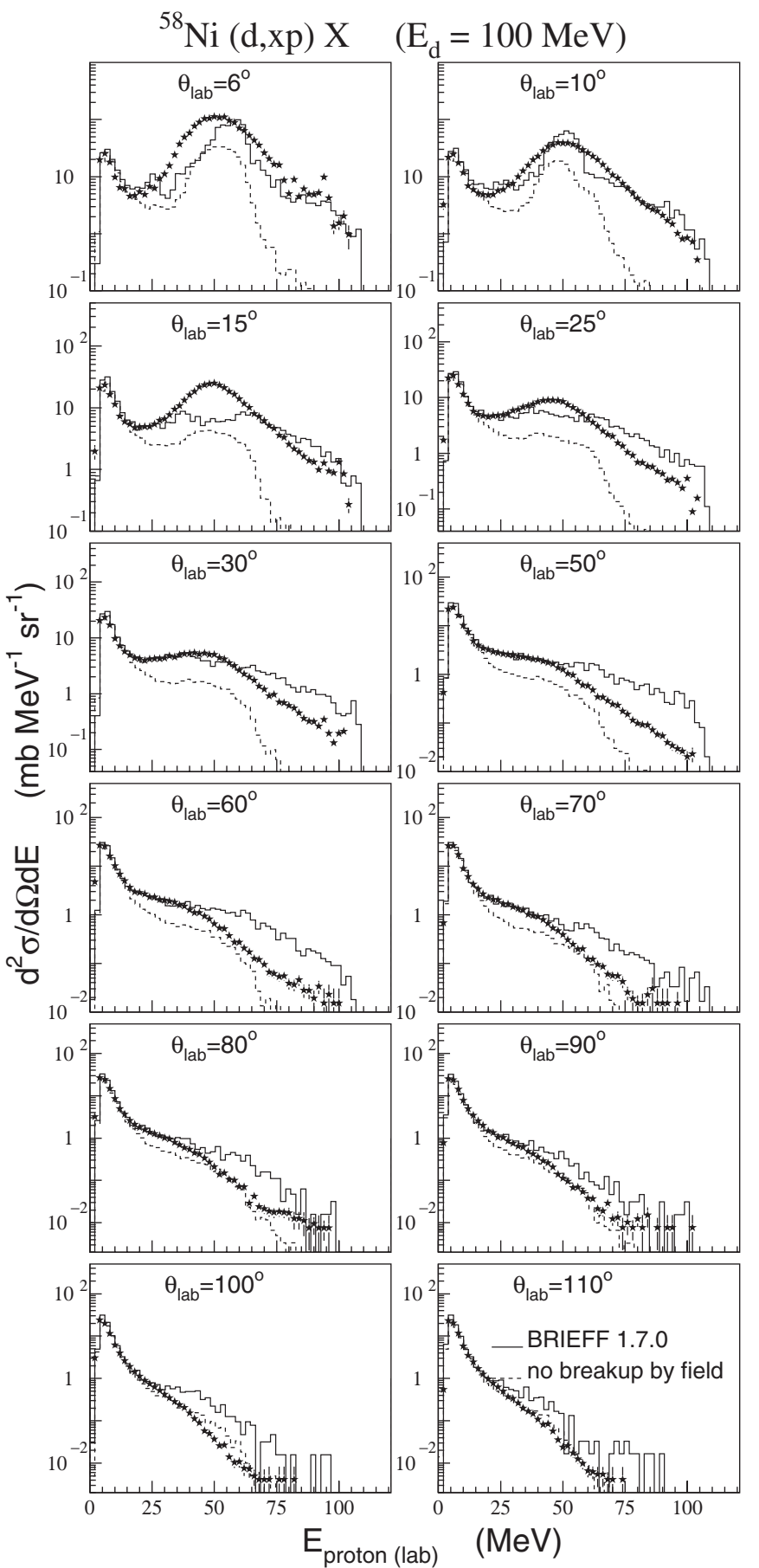

Fig. 1. Double differential cross sections of proton production for $d+{ }^{208} \mathrm{Ni}$ reaction at $100 \mathrm{MeV}$. The solid and hashed histograms are the results of BRIEFF with and without the breakup by the nuclear+Coulomb field, respectively. The black symbols are the data of Ridikas et al. [7]. 


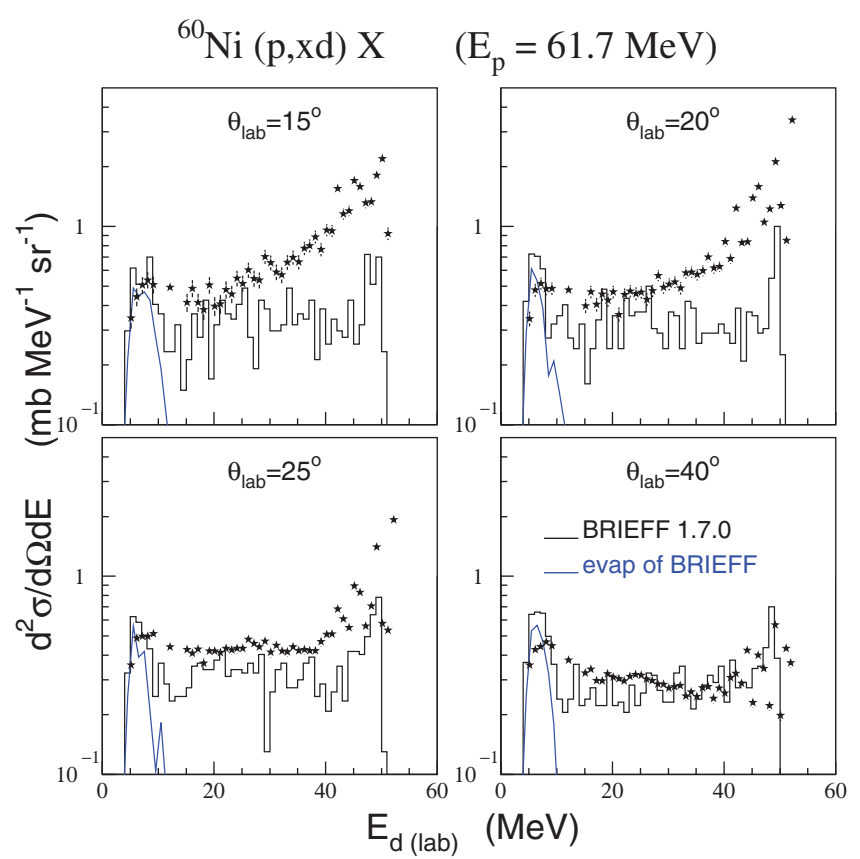

Fig. 2. Double differential cross sections of deuteron production in $p+{ }^{60} \mathrm{Ni}$ reaction at $61.7 \mathrm{MeV}$. Black histograms are the results of the full calculation of BRIEFF and includes deexcitation component (blue lines). The black symbols are the data of Bertrand and Peele [8].

in Ref. [3]. However we noticed a deformation of the deuteron density with time when the equations of motion are applied to the positions $\mathbf{r}^{*}$ and energy-momenta $\left(E^{*}, \mathbf{p}^{*}\right)$ of the neutron $n_{(d)}$ and proton $p_{(d)}$ of the deuteron in its center of mass. To correct this discrepancy the positions of the two nucleons were fixed in the deuteron center-of-mass before the deuteron breakup.

In the deuteron center-of-mass, each nucleon of deuteron has a total energy equal to $\left(p^{2}\left(r^{*}\right)+\right.$ $\left.m_{\text {eff }}^{2}\left(r^{*}\right)\right)^{1 / 2}=m_{N}+e_{\text {bind }} / 2$ where $m_{e f f}\left(r^{*}\right)=m_{N}+V_{d}^{*}\left(r^{*}\right)$ is the effective mass and $e_{\text {bind }}$ is the deuteron binding energy.

\subsection{Deuteron breakup in the nuclear+Coulomb field}

The figure 1 shows the energy-angle distribution of protons in the continuum when the nuclear+ Coulomb breakup is "on" (solid histograms) and "off” (hashed histograms). When nuclear+Coulomb breakup is "off", the deuteron can breakup only if one of the nucleons of the deuteron collide a nucleon of the target nucleus according to the standard collision term of the INC model. When nuclear+Coulomb breakup is "on", the proton-neutron bound system of the incident deuteron can be broken if the difference of the potentials seen by each of the two nucleons in the nucleus frame is greater than an phenomelogical value that depends on the mass of the target nucleus.

The deuteron motion and the condition of deuteron breakup are applied to all candidate deuterons at each time step of the INC. A process to form a quasi-free deuteron from a proton-neutron pair in $\mathrm{BRIC}$ is required to calculate the emission of high energy deuterons.

\subsection{Deuteron formation in nuclear reactions}

A deuteron candidate is created from a pair of quasi-free neutron and proton $p+n \rightarrow d_{\text {cand }}^{(q f)}$ by the following quasi-free coalescence process. The positions and the energy-momenta of the two nucleons 


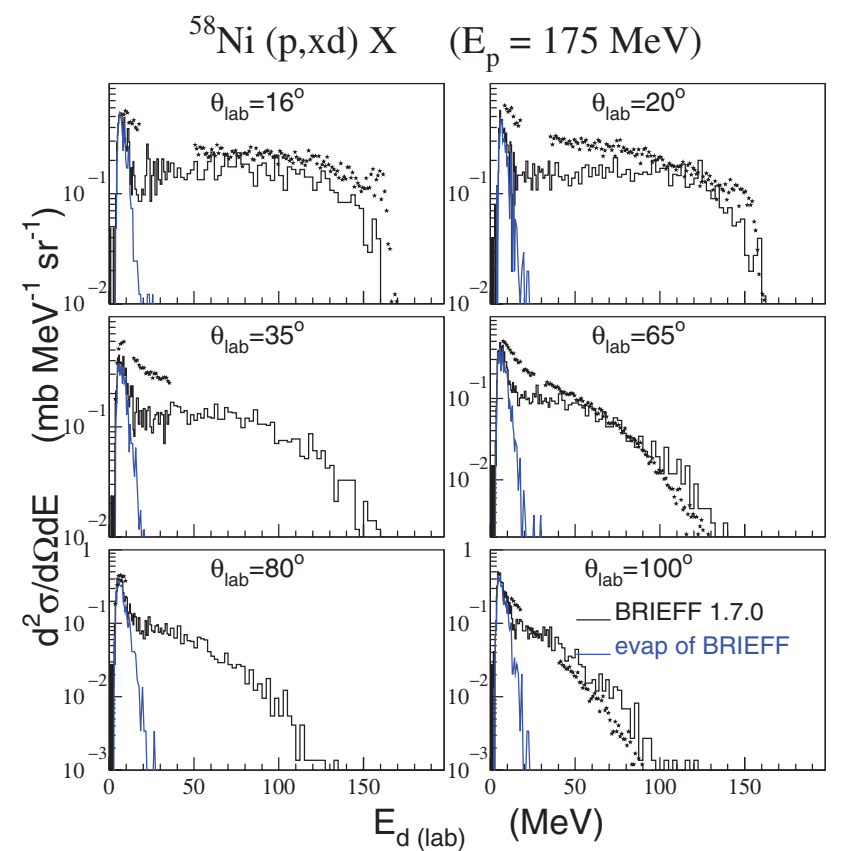

Fig. 3. Same as in fig. 2 for $p+{ }^{58} \mathrm{Ni}$ reaction at $175 \mathrm{MeV}$. The black symbols are the data of Piskor-Ignatowicz et al. [9].

in the center-of-mass frame of the deuteron candidate are deduced from their positions and energymomenta in the laboratory frame with the opposite procedure used in the breakup process. The inverse Lorentz transformation needed in this procedure requires to use the effective mass of the nucleons that depends on the inner potential of the deuteron $V_{d}^{*}$ as if they were inside a deuteron. If the proton and neutron form a good deuteron candidate their relative momentum in the center of mass $\left|\Delta \mathbf{P}_{n p}^{*}\right|=$ $\left|\mathbf{P}_{n}^{*}-\mathbf{P}_{p}^{*}\right|$ should then be equal to $0 \mathrm{MeV} / \mathrm{c}$. Due to numerical uncertainties and model approximations, such value of $\left|\Delta \mathbf{P}_{n p}^{*}\right|$ can not be reached then a cut-off value of $20 \mathrm{MeV} / \mathrm{c}$ is applied on $\left|\Delta \mathbf{P}_{n p}^{*}\right|$ to select good deuteron candidates. Moreover the relative distance between proton and neutron in the centerof-mass frame can not exceed $2 R_{d}^{(\max )}$ where $R_{d}^{(\max )}$ is the maximal radius of the deuteron equal to 6 $\mathrm{fm}$ in BRIC. To have some confidence in this selection method the quasi-free coalescence process was shutdown ("off") and the behavior of quasi-free proton-neutron pairs was studied. The proton-neutron pairs that would form good deuteron candidates fulfilled these conditions during several time steps of INC. When the quasi-free coalescence process and the nuclear+Coulomb breakup are both "on", the deuteron can break up at one time step and form again at the next time step and so on.

A quasi-free deuteron is emitted when the distance between its center-of-mass and the center-ofmass of the target nucleus is greater than $R_{d}^{(\text {max })}+R_{\text {targ }}^{(\text {limit) }}$ (where $R_{\text {targ }}^{(\text {limit })}$ is the radius of the target nucleus defined in [1]) which is above the position of the maximum of the nuclear+Coulomb barrier of emitted deuteron in the target nucleus.

The formation of deuteron can occur at any time step of INC stage and inside the target nucleus in our model, not only at the end of the INC event or when the proton and neutron leave the nucleus as it is often done in other coalescence models (see [5],[6] for examples). Moreover the deuteron can propagate into the nuclear medium by successive breakup in nuclear+Coulomb field and formation processes. The emission of a quasi-free deuteron is then the result of the competition between the deuteron formation and the deuteron breakup by nuclear+Coulomb field or by the INC collision term. 
EFNUDAT Workshop “Measurements and Models of Nuclear Reactions", Paris, 2010

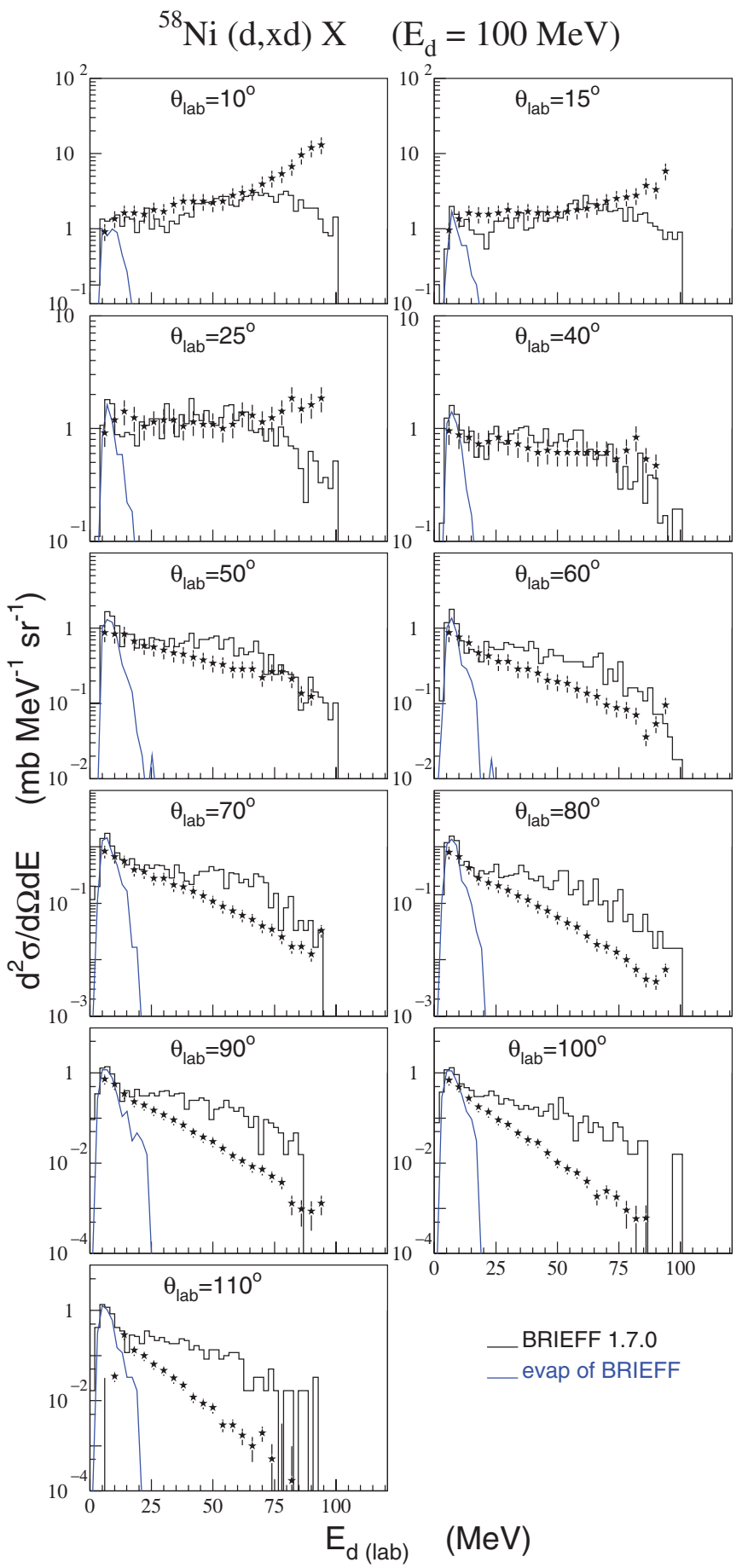

Fig. 4. Double differential cross sections $(d d x s)$ of deuteron production in $d+{ }^{58} \mathrm{Ni}$ reaction at $100 \mathrm{MeV}$. Black histograms are the results of the full calculation of BRIEFF and includes the evaporation component (blue lines). The black symbols are the digitized data of Förtsch et al. [10]. The $d d x s$ values of experimental data were taken at the middle of the data bins with an error of $25 \%$ for digitization error and $10^{-5} / \sqrt{d d x s}$ for statistical error. 


\subsubsection{Results at low intermediate energy}

Figures 2 and 3 show the results of the double differential cross sections of deuteron production in proton induced reactions. At intermediate angles the spectra are in rather good agreement with data of Bertrand and Peele at $62 \mathrm{MeV}$ [8] and with data of Piskor-Ignatowicz et al. at $175 \mathrm{MeV}$ [9]. However the deuteron production of higher energy in forward direction is underestimated by our non-elastic reaction code. This discrepancy comes probably from a poor description of the direct process $p+{ }_{Z}^{A} X \rightarrow$ $d+{ }_{Z}^{A-1} X$ in our model. In the $175 \mathrm{MeV}$ proton induced reaction, BRIEFF gives the overall trend of the energy-angle distribution of outgoing deuterons despite an underestimation of deuteron production cross sections below $60 \mathrm{MeV}$ outgoing energy.

\subsubsection{Results of $(\mathrm{d}, \mathrm{xd})$}

The breakup of deuteron and the formation of deuteron along the INC stage allow to calculate the $(\mathrm{d}, \mathrm{xd})$ energy-angle distribution in the full energy range of the outgoing deuteron. Figure 4 shows the comparison of such results for the $d+{ }^{58} \mathrm{Ni}$ reaction at $100 \mathrm{MeV}$ with the data of Förtsch et al. [10]. The data errors are estimations of our digitization error and of statistical error. The double differential cross sections $(\mathrm{d}, \mathrm{xp})$ of figure 1 and $(\mathrm{d}, \mathrm{xd})$ of figure 4 come from the same BRIEFF calculation of $d+{ }^{58} \mathrm{Ni}$ reaction at $100 \mathrm{MeV}$. The results of $(\mathrm{d}, \mathrm{xd})$ calculation get worse as the detection angle of the deuteron in the laboratory frame increases. Nevertheless we expect some improvement of the $(\mathrm{d}, \mathrm{xd})$ results in backward direction when the formation of ${ }^{3} \mathrm{H}$ and ${ }^{3} \mathrm{He}$ in the INC stage will be fully implementated in BRIC since a small part of the deuteron candidates should enter in the ${ }^{3} \mathrm{H}$ and ${ }^{3} \mathrm{He}$ formation and emission.

\section{Conclusions}

In comparison to a previous work, the breakup of incident deuteron by the nuclear+Coulomb field of the target nucleus has been improved in the INC stage of BRIEFF.

While the production of deuterons was limited to the evaporation component in the previous versions of BRIEFF, the INC stage now computes also their production in the full range of outgoing energy in the nucleon and deuteron induced reactions. In our INC model a quasi-free proton-neutron pair forms a good candidate deuteron if they fulfill the opposite conditions of kinematics to those of the breakup process. This quasi-free coalescence process $p+n \rightarrow d_{\text {cand }}^{(q f)}$ and the deuteron breakup $d_{\text {cand }}^{(q f)} \rightarrow p+n$ are in competition along the time evolution of each INC event and the deuteron production in the continuum at the end of INC stage is the result of this competition.

The production of other light-mass ions $\left(\mathrm{t},{ }^{3} \mathrm{He}, \alpha\right.$-particle, ...) in continuum by our INC code is under way in an approach consistent with the light-mass ions induced reactions.

\section{References}

1. H. Duarte, in Proceedings of the International Conference on Nuclear Data for Science and Technology, April 22-27, 2007, Nice, France, ed. O. Bersillon, F. Gunsing, E. Bauge, R. Jacqmin, and S. Leray, EDP Sciences, 2008, pp 1117-1120.

2. H. Duarte, Phys. Rev. C75, 024611 (2007).

3. H. Duarte, in Proceedings of the 12th International Conference on Nuclear Reaction Mechanisms June 15-19, 2009, Villa Monastero, Varenna, Italy, ed. by F. Cerutti and A. Ferrari, CERNProceedings-2010-001, pp 355-362.

4. R.A. Malfliet and J.A. Tjon, Nucl. Phys. A127, 161 (1969).

5. D.H. Boal, Phys. Rev. C25, 3068 (1982). S.G. Mashnik, Nucl. Phys. A568, 703 (1994).

6. A. Boudard, J. Cugnon, S. Leray and C. Volant, Nucl. Phys. A740, 195 (2004). 
EFNUDAT Workshop “Measurements and Models of Nuclear Reactions", Paris, 2010

7. D. Ridikas, W. Mittig, H. Savajols, P. Roussel-Chomaz, S.V. Förtsch, J.J. Lawrie, and G.F. Steyn, Phys. Rev. C63, 014610 (2000).

8. F.E. Bertrand and R.W. Peele, Phys. Rev. C8, 1045 (1973).

9. B. Piskor-Ignatowicz et al., EXFOR D0580003.

10. S.V. Förtsch, D. Ridikas, W. Mittig, H. Savajols, P. Roussel-Chomaz, G.F. Steyn and J.J. Lawrie, in Proceedings of the International Conference on Nuclear Data for Science and Technology, October 7-12, 2001, Tsukuba, Ibaraki, Japan, J. Nucl. Sci. Technol., Supp 2, pp 792-794 (August 2002). 